\title{
A Novel Image Change Detection Model and Optimization Algorithm based on Game Theory under the Bounded Rationality Conditions
}

\author{
Ning $\mathrm{Wu}^{1}$, Harry Scott ${ }^{2}$ \\ ${ }^{1}$ Jilin Radio and TV University, Jilin, China; \\ ${ }^{2}$ University of Massachusetts, Massachusetts, USA
}

\begin{abstract}
In this paper, we propose novel image change detection model and optimization algorithm based on game theory under the bounded rationality conditions. In the field of remote sensing image application, change detection is of the same area is analyzed by two different periods of remote sensing images, testing to determine the region in a period of change information. To enhance the traditional change detection algorithms we modify the method as the follows. Firstly, we analyze the mathematical forms of the game theory as the co-evolution has the basic characteristics of game theory and the dynamic characteristics of co-evolution through continuous evolution and eventually reaches a stable equilibrium state because of the feature. Later, we discuss the novel mathematical optimization approaches for the change detection based on the proposed game theory prior. Then, we combine independent component analysis and the bounded rationality conditions to finalize the detection algorithm. The independent component analysis is a data processing method appeared in recent years which can be as an extension of PCA and it will be the data transform into each other on the direction of the independence with higher robustness. In the experiment part, we simulate the experiment with the other state-of-the-art algorithms. The result reflects that our algorithm outperforms under various conditions and enhances the detection accuracy from $95.8 \%$ to $97.9 \%$ which has made the new breakthrough.
\end{abstract}

Keywords: Image Change Detection; Image Processing; Mathematical Optimization; Game Theory; Bounded Rationality Conditions; Ground Truth; Contrast Test.

\section{Introduction}

Change detection is based on the same area at different times of the comparison of two or more remote sensing image analysis, and the differences between image features change to get the required information. Because the landscape is affected by the general changes in the environment and human activities have been changing, effectively monitoring the change information, analyzing the characteristics of the dynamic change and reason and resource management department of environmental protection is an urgent need to solve the problem, and remote sensing image change detection is monitoring and dynamic change information is an effective method. It can use the computer to the same area at different times of comparison and analysis of remote sensing images and get the feature change information to make people able to be quickly focused in the area of interest, so as to improve the efficiency of the interpretation of remote sensing image interpretation [1-2]. Remote sensing image processing and the understanding of the content, mainly including the remote sensing image registration, correction, de-noising, edge detection, classification, segmentation, feature extraction, object recognition and fusion processing, and change detection, etc. By studying the content, finally can meet the demand of automatic interpretation of remote sensing images. Among them, the remote sensing image can provide large range of dynamic observation data, has the continuity of 
the sequence of features in space and time, so long the phase image data can be used in the monitoring and feature perception study. The change detection technology is one of the main applications of remote sensing technology [3]. In the following figure one we demonstrate the example of the remote sensing change detection task's applications.
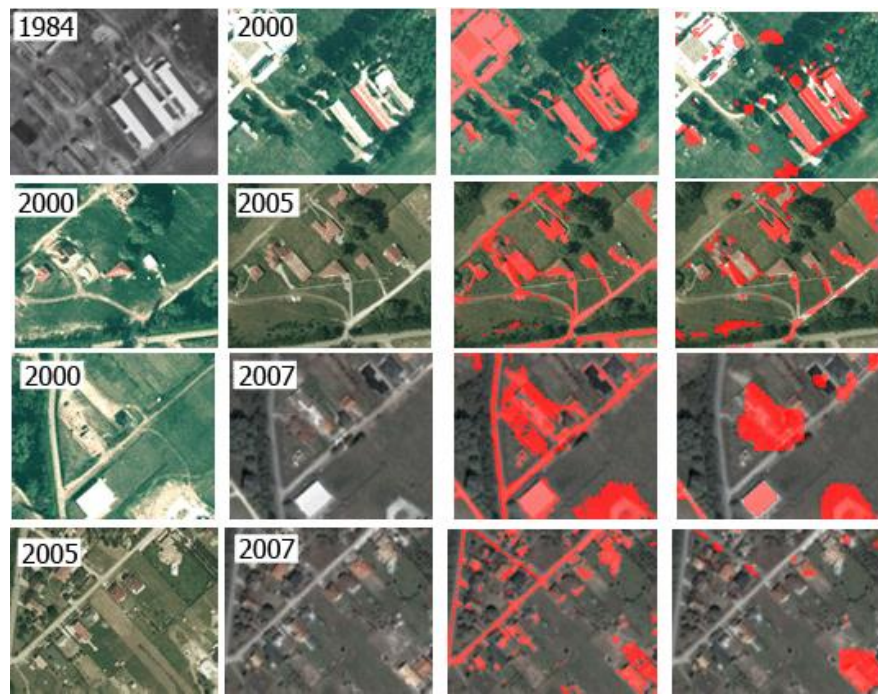

Figure 1. The Example of the Remote Sensing Image Detection Task

The existing change detection methodologies could be summarized as the following parts. (1) The image difference method. This test method is the most easy to understand, it will not phase image at the same time, in accordance with the band of wavelengths like the object way difference one by one, get a new pair of difference image, then analyze the difference image processing. Usually for the precise registration of homologous remote sensing image effect is very good also [4]. (2) The correlation coefficient method. Correlation coefficient method to calculate time of corresponding pixel in image correlation coefficient, the results of represents the two times corresponding to the correlation of pixels in the image. Usually take window, computation of the two images corresponding to the correlation coefficient of the windows to show the correlation of window center pixel [5-6]. (3) Image ratio method. This kind of the processing method and differential method are very similar, and is suitable for extracting the brightness change of absolute value is not big, but with the original brightness the proportion of relatively large changes in the value. Changes in and did not like the division of when use experience to determine methods lack of mature and effective change threshold determination method. (4) Image regression methodology. Threshold method to determine areas for change after dealing with the return of the remote sensing data to a certain extent, like dried radiation correction of the relative correction, so this can weaken long phase of data due to atmospheric conditions and adverse effect brought by the different angle of the sun, also can be seen that pretreatment of radiation correction for the effect of this method is not very big. (5) Spectral characteristic variation method. Multi-band remote sensing data fusion in the different source, reflex response curves of same object should be consistent, but if information is inconsistent, then after fusion image spectrum act is different from normal feature and at this time is called the spectral characteristic variation features and these features town in order to pass the image interpretation drew out. (6) Change vector analysis method. Spectral change vector analysis method is based on not change by the radiation between the phase images at the same time, analyzes the differences of each band, determine the strength and direction of change. To the remote sensing images of different time, the image spectrum measurement, each like the pixel can generate a change direction and strength change of two feature vectors [7-8]. In the 
figure two, we demonstrate the change detection procedure from the algorithmic perspective.

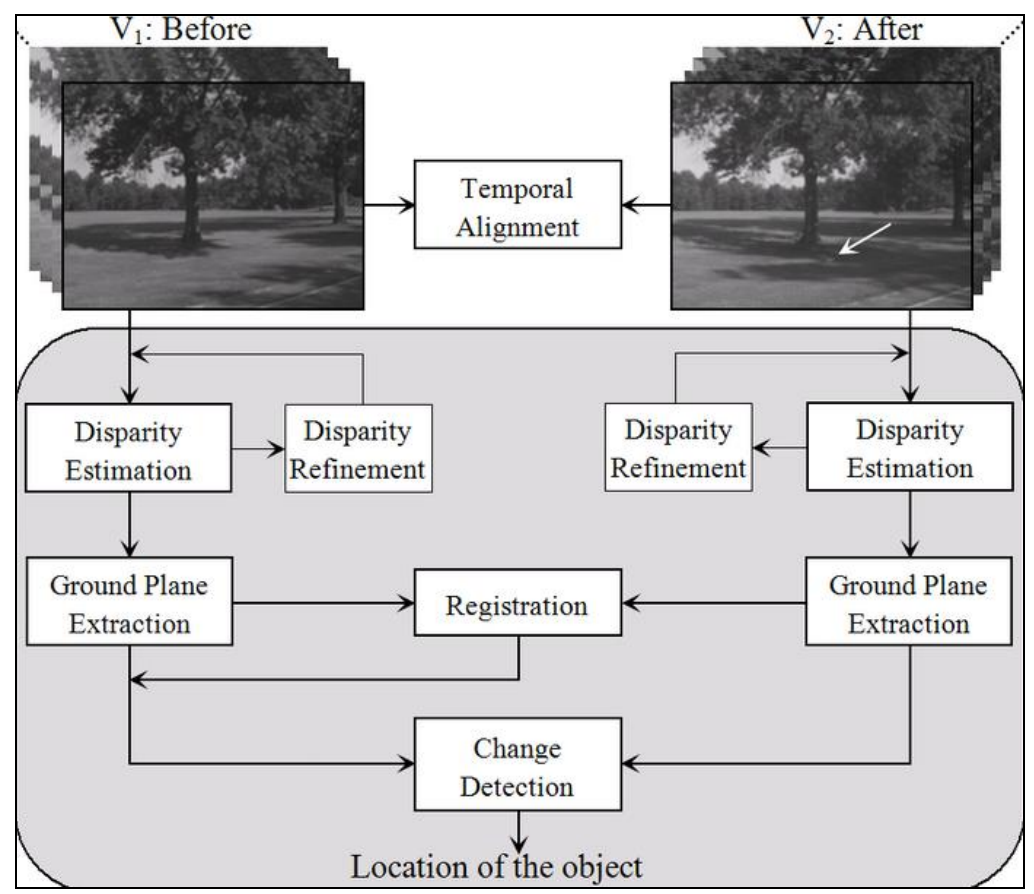

Figure 2. The Algorithmic Description of the Image Change Detection Procedure

Game theory is a mathematical method is used to study the nature of the confrontation or the cooperation in the competition and it is an important branch of operational research. The previous mathematics is established for the development of physics and it describes the main behavior is the objective world and is not subjective economic activity, the object of study is more like a game of the participants, between each other, which describes the object of study need different mathematical tool, the new mathematical tool, is the game theory [9-11]. In the game theory, both for complete information game or for the incomplete of information game, it has a very standard definition. Complete information game are defined as follows: under the given any strategy combination, if all game players can clear any other game participants' benefits, then this game is the complete information game. If we can't clear the other game participants' benefits, even if the only one game participants' benefits is not clear, the game is incomplete information game, not clear here refers to the participants subjective judgment and think that the benefits of other participants may have the possibility of the variety of the unknown. We will adopt the concept of the game theory in the later discussion.

The rest of this paper is organized as the follows. In the section two, we review the recent proposed novel algorithms for the image change detection which will serve as introduction of our work. In the section three, we analyze the principles of the game theory and the potential application points for our research. In the section four, we analyze the optimization approach based on the mathematical analysis for the later discussion. In the section five, we propose the algorithm of our researched with the theoretical analysis and discussion. In the section six, we conduct theoretical simulation on the proposed algorithm compared with the others. Finally, we conclude the research and settle down the future research orientation. 


\section{Literature Review and Analysis}

In this part, we review the latest research on the topic of the image change detection. In [12], Txomin Hermosilla proposed novel research with annual gap-free surface reflectance composites. In their research, the take different classification model, clustering of difference image segmentation, expects to get the optimal global threshold, which will change with the regional division. That paper proposes a quantum immune clonal clustering algorithm based on the change detection methods to achieve the change detection with clustering, the change detection problem of area as combinatorial optimization problem. In [13], Peijun Du conducts research on the hierarchical unsupervised change detection in multi-temporal hyperspectral images. A multi-band remote sensing image change detection method based on MRF using the MRF model integration difference change information of the image of each band. Method overcome the deficiency of the traditional change detection method it can obtain better testing information, the result can be for land use cover change detection, environmental testing and the other fields to provide decision support. In [14], Bruzzone, L. conducts research on the very high resolution image change detection. They proposed that images regularly acquired over urban areas opens new attractive opportunities for monitoring human settlements at the level of the individual buildings. In [15], Haoxiang conduct research on the image restoration and change detection based on game theory. They pointed out that restoration of images plays a de-noising role due to complex movement while obtaining a SAR image. The segmentation procedure transfers the difference map into change map. In [16] Lifeng Lai proposed research on byzantine fault tolerant of the distributed quickest change detection. They introduce and solve the problem of Byzantine fault tolerant distributed quickest change detection in both continuous and discrete-time setups. More reviews could be found in [17-25].

\section{The Game Theory and the Applications}

For a game, the games of the participants are called players, like the game player, usually set by the $\mathrm{N}$ players. A game should have at least two players, or otherwise will not be able to constitute a game. In any game, each player at least has more than one strategy, as well as the ability to make decisions for strategy. The reason is that when a player is only one strategy, regardless of the opponent's strategy change, he can effectively make the "suspense" option. The ability to make decisions for the strategy means players can completely and choose the strategy, not forced or involuntary selection strategy. In the formula one, we define strategy of game where the $P_{n}$ denotes the payoff function [26-27].

$$
G=\left\{P_{1}, P_{2}, \ldots, P_{n} ; u_{1}, u_{2}, \ldots, u_{n}\right\}
$$

Nashville equilibrium refers to a strategy portfolio as all of the participants faced such a situation, when others don't change strategy, his strategy is optimal.

$$
u_{i}\left(P_{i}^{*}, \mathrm{P}_{-I}\right)>u_{i}\left(P_{i}, P_{-i}\right), \quad \forall P_{-i}, \forall i \in N
$$

The optimal dynamic and based on the evolution of the replication dynamic response is the most commonly used two kinds of the dynamic decision-making mechanism. The optimal of reaction dynamic mechanism is generally used when game rational degree is higher, have fast learning ability and when the game people rational degree is low or involves the collective decisions, is generally the replication dynamic mechanism of biological evolution simulation game studies and dynamic adjustment strategy. The formula 3 defines the process.

$$
x^{\prime}=x\left(f-f_{0}\right)
$$


In the formula three, the $f$ denotes average fitness and adapt to the function. By the above theory, we propose the following definitions.

$$
\varepsilon_{i}\left(p_{-i}\right)=\left\{\arg \operatorname{maxu}_{i}\left(p_{i}, p_{-i}\right), p_{-i} \in P_{-i}\right\}
$$

$\varepsilon_{i}$ is represented as the possession of strategy. In other words, if a selection strategy of the $\mathrm{S}$ less than the population average fitness, the fitness of the individual well selection strategy $S$ the proportion of individuals in a group of will as the evolution of time and decreasing. The optimal policy set and Nash equilibrium is the same. So the strategy is a pure strategy Nash equilibrium, at this time of every participant to meet:

$$
U_{i}\left(\tau_{i}, \tau_{-i}^{*}\right) \leq U_{i}\left(\tau_{i}^{*}, \tau_{-i}^{*}\right)
$$

Evolution as an extract from the phenomenon of life important adaptive mechanism has to common understanding and the widely used, but the evolution of the existing model of a common deficiencies is not very well reflect the common facts. In game theory and artificial intelligence in the process of common development, a key problem is how to establish the mathematical model based on game theory, the mathematical model to abstract the conducive to the interests of the game using the contradictions, as well as the interests of both sides, so as to construct the game subject, thus introducing the game theory [28-29].

$>$ Bureau of nature is the natural formation of the main a game and it is determined by bureau of nature's profit function. How to construct natural player revenue function and corresponding policy set has become a key factor influence efficiency of game.

$>$ Virtual players is main person build a game and the interests of the corresponding are required by the optimization goal, it is decided by natural players work together. This means that virtual players can only passive choice whether to accept the strategy.

The above principles denote the primary standards for the performance modification. To achieve the optimal solution for the issues, we should obey the following rule.

$$
F_{\text {optimal }}(t+1) \leq F_{\text {optimal }}(t)
$$

From a mathematical point of view, the co-evolution has the characteristics of game theory and the dynamic characteristics of co-evolution through continuous evolution and eventually reaches a stable equilibrium state because of the characteristic of the problem domain and the steady state is the optimal solution. Assuming that co-evolution algorithm can be represented as a matrix game, as the optimization solution of the game, eventually reach the equilibrium of co-evolution algorithm. Because the game system is completely consistent, when the natural state of the players for unrelated to each other, each natural innings per capita can maximize their own interests and don't need to care about other bureau of nature. When the natural players linked to each other, means that the bureau of nature contain each other, each other need natural bureau middleman layer added consultation mechanism and let bureau of nature to consult each other, draw up each strategy after the bureau of nature are acceptable, and entered the game, game with virtual players. The return matrix is defined below [30].

$$
\left[\begin{array}{cc}
\left(s_{i}-\text { up }, t-u p\right) & \left(s_{i}-\text { up }, t-\text { equality }\right) \\
\left(s_{i}-\text { down, } t-u p\right) & \left(s_{i}-\text { equality, } t-\text { equality }\right)
\end{array}\right]
$$


Through the multiple has its own problem of the solving ability and behavior target agents collaborate to achieve the goal of solving complex, large-scale problems. Each of agent may have different objectives, system of overall goal is implemented in the process of various sub-targets synergy, such multiple agents with different target need to its target, and so on carries on the reasonable arrangement of resources to coordinate their behavior. In the following figure three, we demonstrate the two types of the typical game procedures.

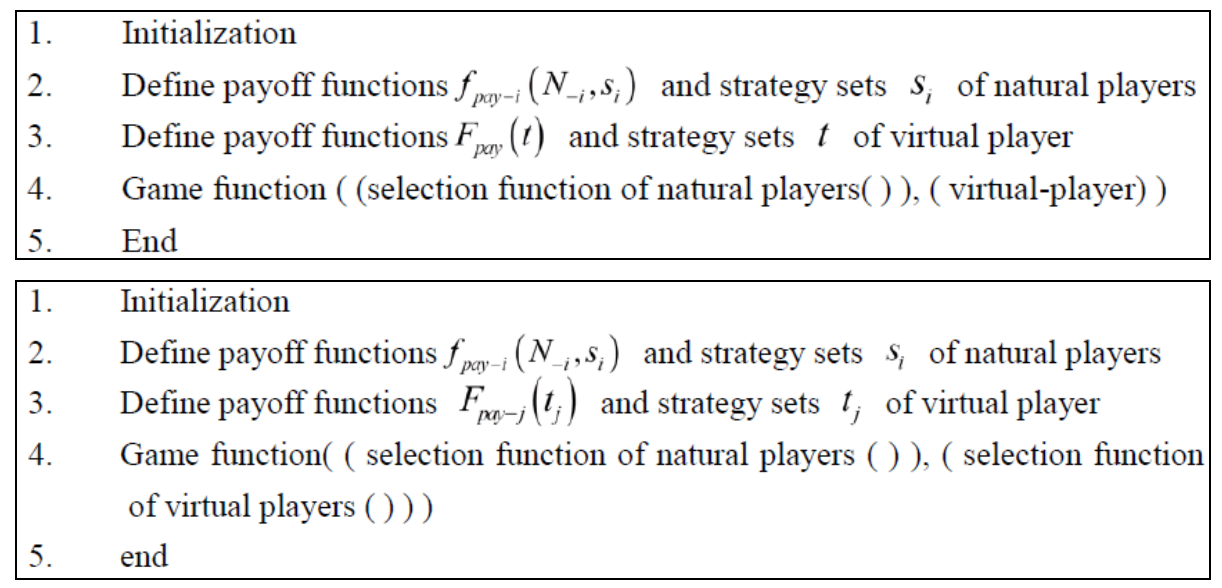

Figure 3.The Two Types of the Typical Game Procedures

\section{Mathematical Optimization for Image Processing Tasks}

For nonnegative constraint is not enough to gain the image which has the invariance said problems, this chapter puts forward a nonnegative matrix decomposition method based on the terrain constraints and the in the process of the decomposing matrix by introducing a terrain constraints optimization code factor, to study the characteristics of the immutability. Due to topographical constraints is a two layer containing square nonlinear and square root of the nonlinear network of bottom-up and its can through will belong to same subject characteristic of the structure of the related pooling together, makes the related characteristics in a structure of lower dimensional subspace in together. To learn with feature invariance image said [31].

Nonnegative constraint is not enough to get the sparse representation and the novel norm of sparse constraint to ensure that the sparse coding matrix is proposed as follows.

$$
O_{\text {Modified }}=\left\|X-W S^{T}\right\|_{F}^{2}+\lambda \sum_{j k} s_{j k}
$$

From the point of view of mathematics, when given a data matrix, to optimize the terrain is network by minimizing the following objective function [32-35].

$$
p=\sqrt{\square+H \times\left(\left(W^{T} X\right) \Theta\left(W^{T} X\right)\right)}
$$

It will belong to the same underlying theme of the structural characteristics of the pooling together, after the forced matrix decomposition of image coding is orderly organization in the same topographic map. Therefore, the sparse coding matrix is modified as follows.

$$
O^{\prime}{ }_{\text {Modified }}=\left\|X-W S^{T}\right\|_{F}^{2}+\lambda p(S)
$$

Compared with the additive noise, on the surface, the degradation model is a little bit more complex, but in the solving process is still very difficult, to solve the model is not 
good. Predecessors' although some classic model is established, a lot of the model is designed to $\log$ and it is involved in the process of solving the image pixel value is negative. Therefore, to get the optimal solution for the objective function, we could rewrite it as the follows [36].

$$
O_{\text {Modified }}^{\prime}=\left\|X-W S^{T}\right\|_{F}^{2}+\lambda p(S)=\operatorname{Tr}\left(\left(X-W S^{T}\right)\left(X-W S^{T}\right)^{T}\right)+\lambda p(S)
$$

Based on the traditional multi-objective and optimization method of mathematics planning principle in the practical engineering optimization problems is often show some vulnerability. The principles of the optimization tasks could be summarized as the follows. (1) The varargs polymerization method. Other of multi-objective optimization evolutionary algorithm is based on the traditional method. In terms of these methods, are aggregated into a multiple objective function with a parameter of single objective optimization functions in different optimization operation, the parameters of the function does not change but at same time in the operation of the change. (2) Switching multiple targets. Not to adapt the multiple targets combined into the single value, this kind of multi-objective optimization evolutionary algorithms in the process of search to switch between the multiple targets. Every time when an individual is selected for reproduction, different target determines which one member of the group is copied into the mating pool. (3) A multi-objective optimization evolutionary algorithm maintains population diversity is very important. But simple genetic algorithm tends to converge to single solution, because the three factors makes the genetic algorithm is often lost some solutions [37-38].

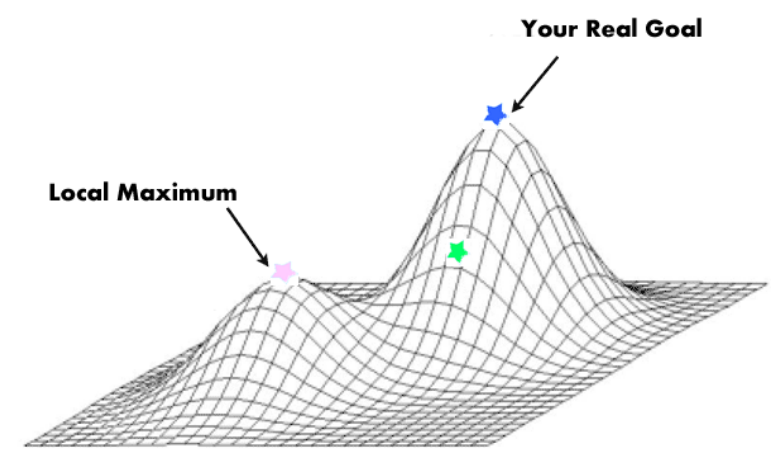

Figure 4.The Illustration of the Local Optimal Solution

As demonstrate in the figure 4, there may be some cases concerning of the local optimal. To deal with the challenge, we adopt multi-objective particle swarm optimization algorithm for general modification. It is a kind of based on iterative optimization tools and the system initialization, a set of random solution by iterative search for the optimal value, not only has good global search capability and has the strong local optimization ability. The mathematical description of the algorithm is as follows. Each particle contains for the position of a multi-dimensional vector and speed vector that denoted as the formula 12.

$$
x_{i}=\left(x_{i 1}, x_{i 2}, \ldots, x_{i D}\right), \quad v_{i}=\left(v_{i 1}, v_{i 2}, \ldots, v_{i D}\right)
$$

Each particle's position and velocity update press type is shown as the follows [39-40].

$$
\left\{\begin{array}{c}
x_{i d}^{t+1}=x_{i d}^{t}+v_{i d}^{t+1} \\
v_{i l}^{t+1}=w v_{i l}^{t}+c_{1} r_{1}\left(p_{i d}^{t}-x_{i d}^{t}\right)+c_{2} r_{2}\left(p_{i d}^{t}-x_{i d}^{t}\right)
\end{array}\right.
$$

The first part is the speed of the original particles, the greater its value, the more conducive to global search and local search capabilities, its value is small is needed for balanced global and local search ability. The second part is a reflection of the particles 
themselves, which indicates that their experience to current search tend to attract. Particle swarm optimization algorithm is a kind of new optimization algorithm, according to its own optimal location and its each particle group global optimal location update their speed and position, the particles because of the influence of the group of the global optimal position, quickly converge to global optimal location nearby, it has shown its rapidity, the effectiveness and robustness, and other advantages.

\section{The Proposed Change Detection Algorithm}

In the field of remote sensing image application, change detection is of the same area is analyzed by two different periods of remote sensing images, testing to determine the region in a period of change information. Change detection based on pixel level is not at the same time after the registration of remote sensing image in pixels, the level of comparative analysis, whether on each like the basic change and the type of change. This kind of algorithm is the directly using the original pixels, information value change detection, is currently the most widely used change detection technology. Due to the image quality and the change detection scene change constantly. Determine the threshold value in practical application is a difficult job and it is difficult for fixed threshold to determine all the test results and some test results of the algorithm is sensitive to the change of threshold value, the slight change could lead to a threshold detection results in the index changing dramatically.

\subsection{The Independent Component Analysis}

In change detection method based on data transformation, the most widely used principal component analysis and it is through the PCA transform to eliminate the long phase of remote sensing image asked the relevant information, so as to make the change information after the transformation of each component to be enhanced images. PCA is a method based on second order statistics, only in the signal statistical distribution accords with Gaussian, to completely eliminate the signal between the relevant information, and for non-Gaussian signals only to remove the second-order correlation between information. The principle of PCA is through the transformation, the transformation of data to a mutual orthogonal direction vector and gets a set of component orthogonal to each other shown as below.

$$
X=U \sum V^{T}
$$

Independent component analysis is a data processing method appeared in recent years, can be as an extension of PCA, it will be the data transform into each other on the direction of the independence, not only requires the orthogonal between each of the component and need to be independent. We define the signal input in the formula 15.

$$
x_{i}=a_{i 1} s_{1}+a_{i 2} s_{2}+\ldots+a_{i n} s_{n}, \quad i=1,2, \ldots, m
$$

The corresponding vector type of the signal could be re-written as the formula 16 .

$$
X=A S=\sum_{j=1}^{n} a_{j} s_{j}
$$

Because of the ICA was applied to the output of the signal separation vector expected the statistically independent nature, so the first thing needs to be defined is to determine its core independence criteria. In determining the independence of the signal components in the standard, the mutual information based on information theory is one of the commonly used methods. Mutual information is defined as the formula 17.

$$
I(y)=\int p(y) \lg \frac{p(y)}{\prod_{k} p_{k}\left(y_{k}\right)} d y
$$


Where the $\prod_{k} p_{k}\left(y_{k}\right)$ represents the joint probability density by solving objective function extremum statistical gradient. In remote sensing images, each image is mixed by a variety of features, if the spectral characteristics of all sorts of the features in line with the Gaussian distribution, we can through use of Gaussian mixture model to simulate the remote sensing images. In the Gaussian mixture model has been widely used in the image processing and analysis, also has obtained the quite ideal effect. In the following figure 5, we illustrate the difference of the PCA and the proposed ICA.

PCA

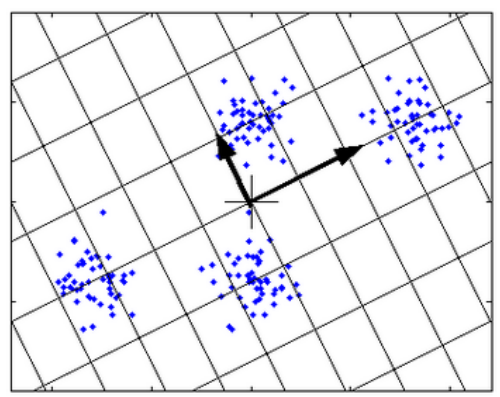

ICA

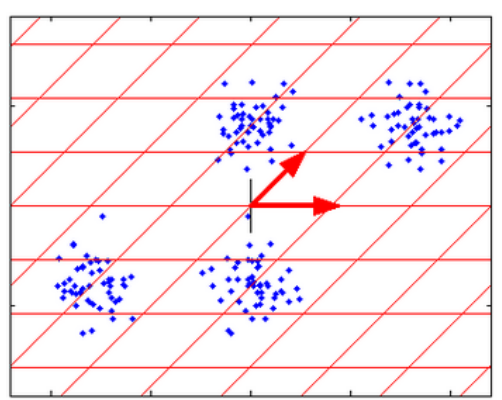

Figure 5.The Differences of the PCA and the Independent Component Analysis

\subsection{The Limited Rational Feature Extraction Algorithm}

After add the entropy of the significant figure will increase, it must change the value of the area and edge consistency is better, because the generally change area the edge of the area, belongs to the gradient area, namely the relative to the prospective area change, change is not obvious, the local entropy can use must change the value of the area, improve the value of the gradient area, therefore, to improve local entropy fuzzy change region detection accuracy. Local entropy reflects the image characteristics of discrete degree, where local entropy is big. The image is relatively uniform, small, local entropy image features large discreteness and it has nothing to do with the image characteristics of the mean, so we can according to the local entropy of image is relatively homogeneous target partition as local entropy is window more pixels joint contribution for the single point is not sensitive to noise. To calculate the overall features and the related saliency, we use the following three features as the measurement.

$$
\begin{aligned}
& f_{1}=\sum_{i} \sum_{j}(i-j)^{2} p(i, j) \\
& f_{2}=\sum_{i} \sum_{j} p(i-j)^{2} \\
& f_{3}=\frac{\left[\sum_{j} \sum_{i}(j i) p(j, i)-u_{x} u_{y}\right]}{\partial_{y} \partial_{x}}
\end{aligned}
$$

If the change amplitude vector, the greater the change intensity, the greater the also showed that pixel spectrum difference is bigger. If the change of the corresponding pixel vector set amplitude exceeded a certain threshold, the pixels as the group has changed. After calculating the variance of the $f_{3}, f_{2}, f_{2}$ and the direction, we could obtain the four directions of the mean and variance and we get six texture feature with rotation invariant properties of texture feature vector. Simple image direct comparison method no use to the spatial information of image, and no considering the impact of noise or light changes and 
it is easy to cause virtual test or inspection. How effectively use the image itself contains the spatial information and asked the useful factors of remote sensing images for phase to be a need to solve the problem.

\subsection{The Core Procedures of the Algorithm}

Each pixels, in the remote sensing images by the corresponding mixture of many kinds of feature information, and each signal source independent of the each other, and some of these signal source is stationary, some of them are change. Constitutes the background image can be thought of as a stationary signal source, and change of signal source constitutes the image change, change the image and the background image is also independent of each other.

Remote sensing images before and after the phase are composition of mixed signal image by the background image and change the mix of two independent signal sources. In the ICA method, mixing way to independent signal source is linear, within the implementation in the form of product. In the KICA, using the inner product in the kernel function instead of ICA, nonlinear transform of data is mapped to high-dimensional feature space for the independent component analysis to reduce the error of the result of the nonlinear mixed spectral.

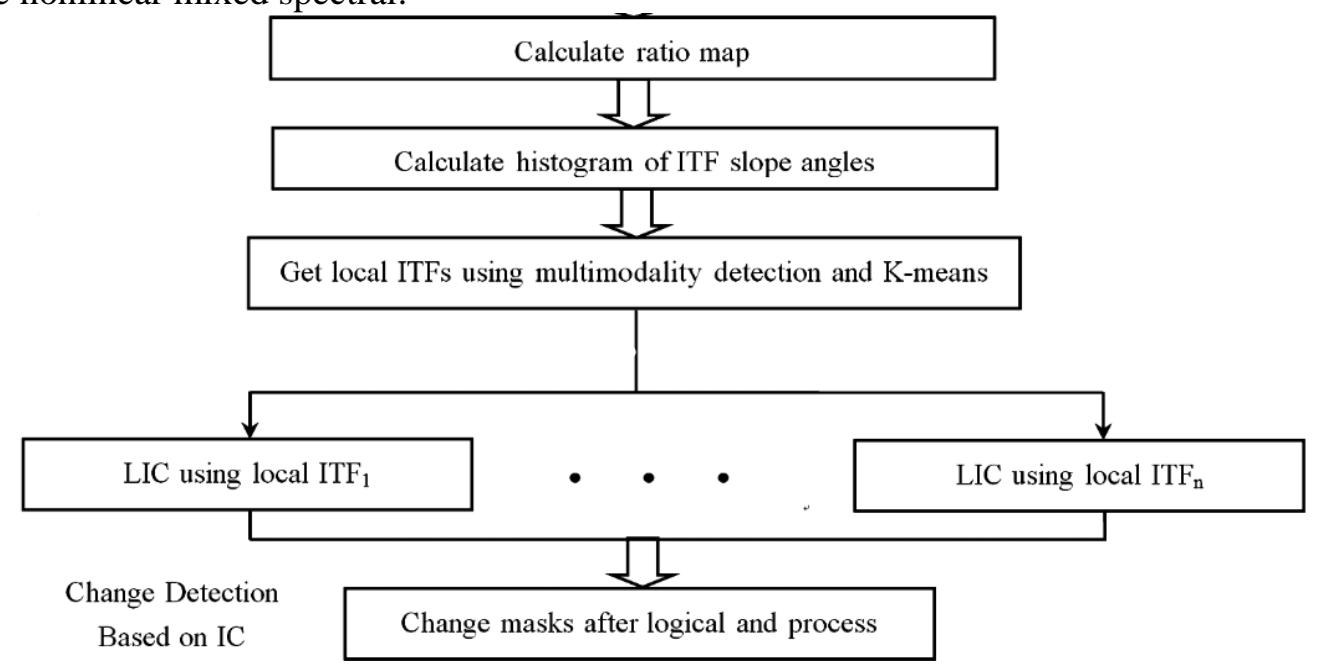

Figure 6.The Main Steps of the Proposed Methodology

\section{The Experiment and Simulation}

In this section, we simulate the proposed algorithm. In the figure 7 , we illustrate the test images and the ground truth reference. In the figure 8 9, we show the two sets of the simulate result that demonstrate the performance of our method compared with other state-of-the-art methods. In the figure 10, we show the change detection accuracy for our algorithm.
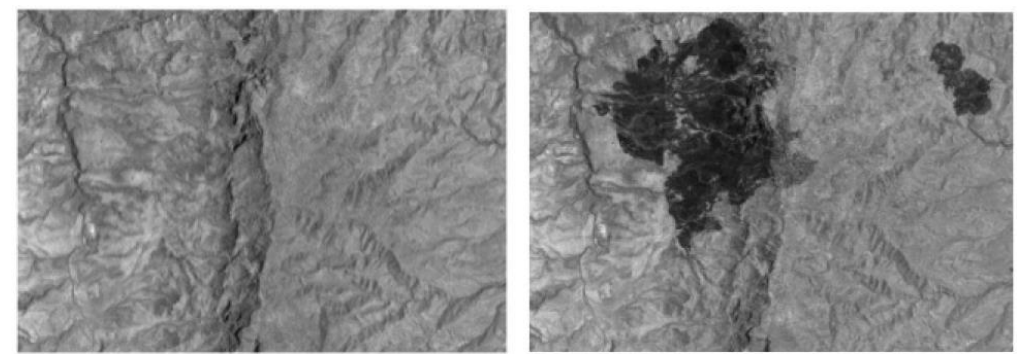

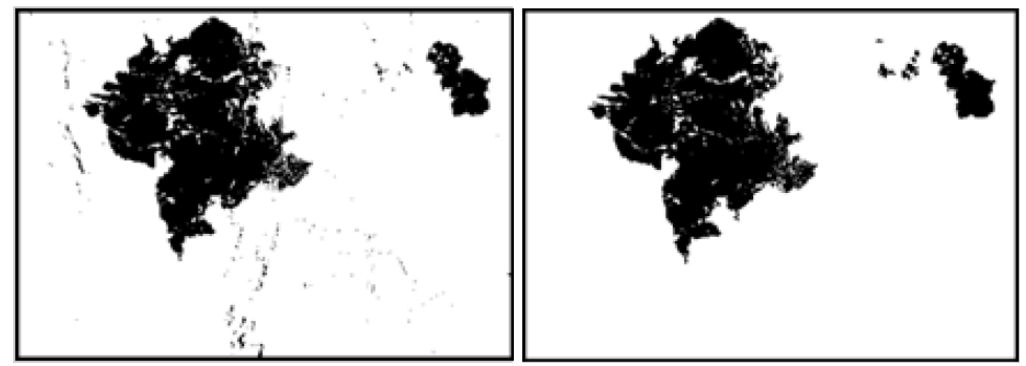

Figure 7.The Testing Images and the Ground Truth References
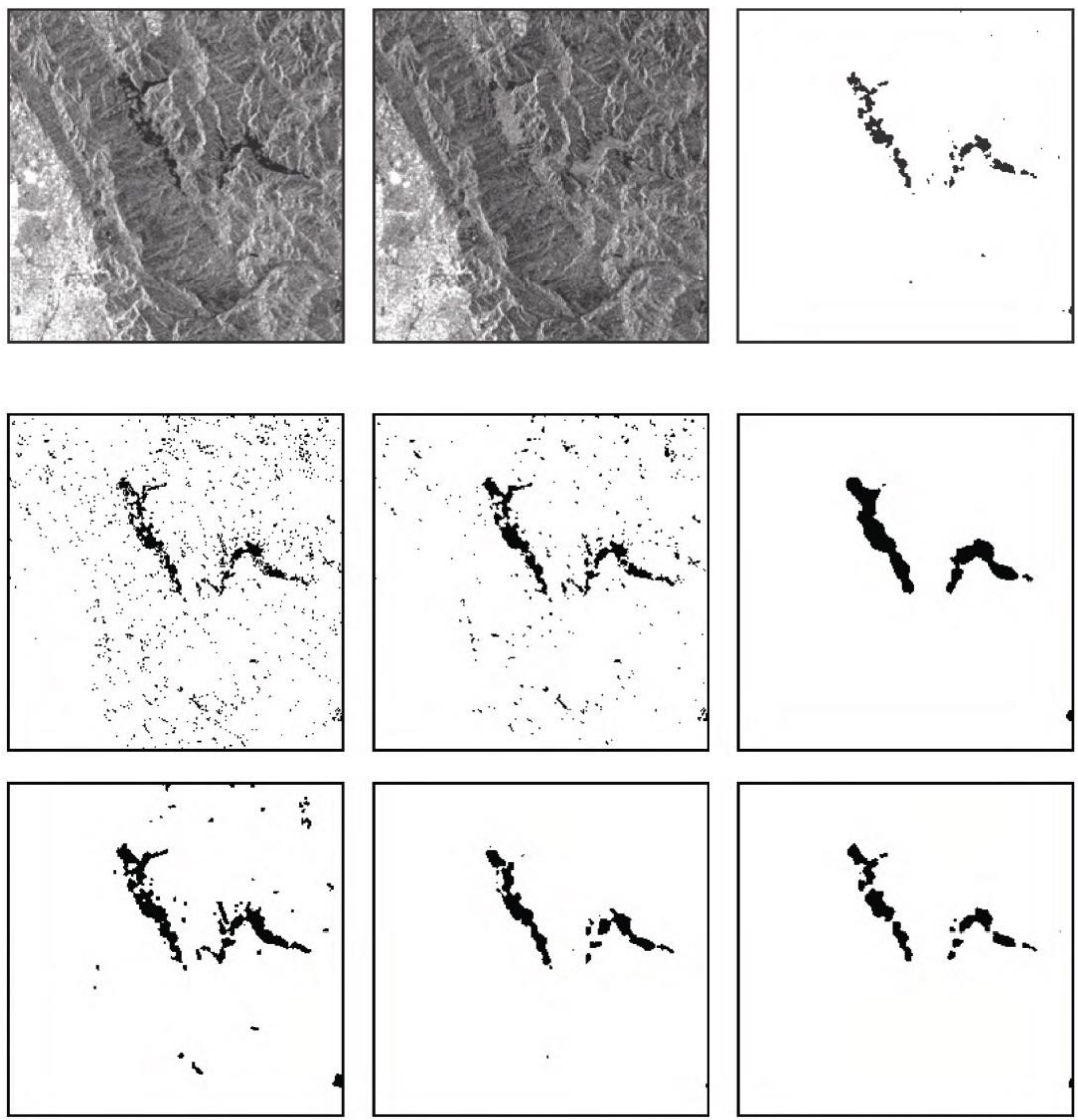

Figure 8.The Comparison Experiment Set One with the Ground Truth
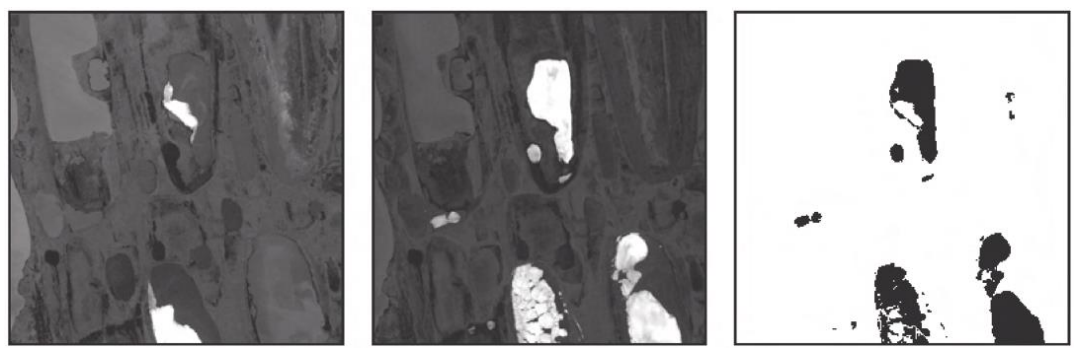

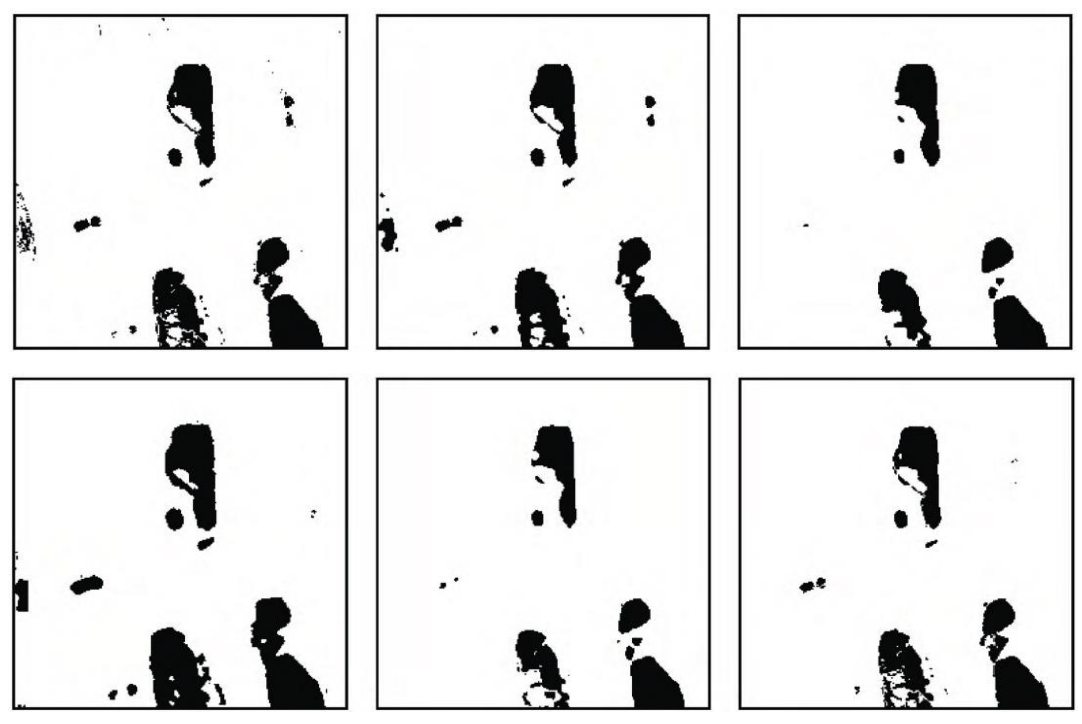

Figure 9.The Comparison Experiment Set Two with the Ground Truth
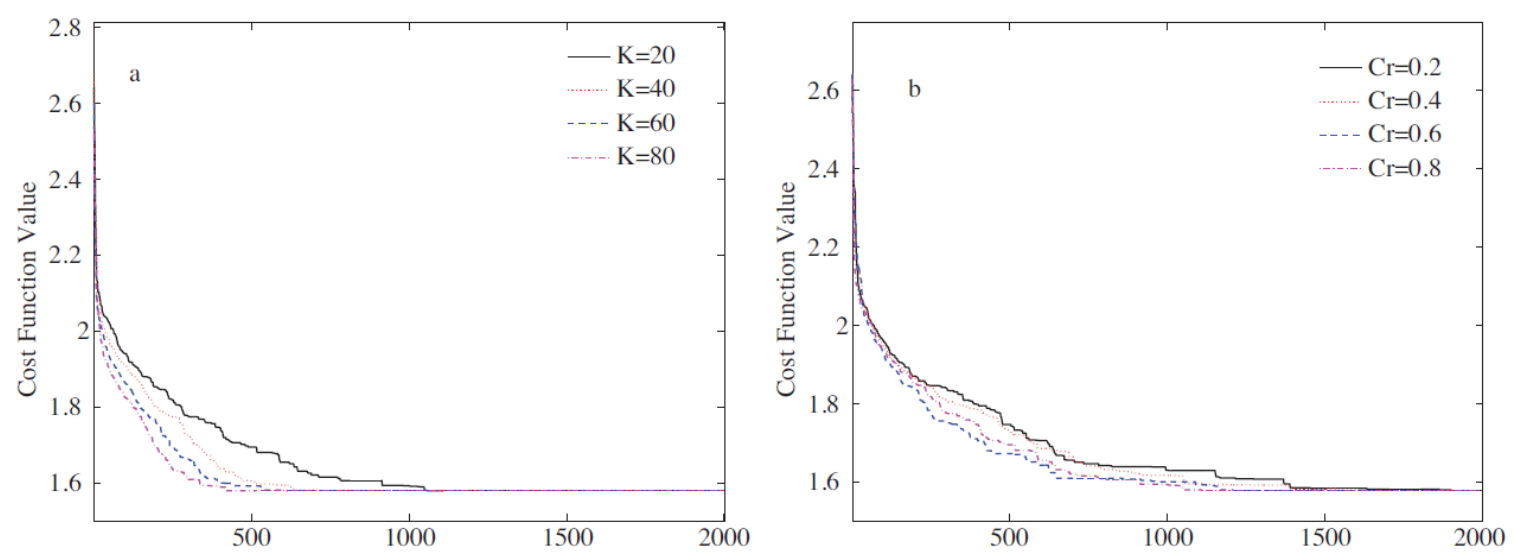

Figure 10.The Change Detection Accuracy Statistical Data Curve

\section{Conclusion}

In this paper, we propose novel image change detection model and optimization algorithm based on game theory under the bounded rationality conditions. In the applications of remote sensing images, remote sensing image change detection is detected in the same area is not at the same time the state of remote sensing images. The remote sensing image change detection has very important role in many fields and provides the scientific decision basis for related departments. Our algorithm through the mixed kernel function, considering the difference of the image grayscale characteristics at the same time considering the difference image texture feature and makes change detection results more robust. Using difference image classification thought to avoid the difference image threshold selection problems. Through the comparative analysis on the experimental data, the feasibility and effectiveness of the proposed algorithm is proved. In the future, we will apply our algorithm into the very high resolution images to test the feasibility of the method under the large data environment. 


\section{References}

[1] Zhu, Zhe, and Curtis E. Woodcock. "Continuous change detection and classification of land cover using all available Landsat data." Remote sensing of Environment 144 (2014), pp. 152-171.

[2] Tewkesbury, Andrew P., Alexis J. Comber, Nicholas J. Tate, Alistair Lamb, and Peter F. Fisher. "A critical synthesis of remotely sensed optical image change detection techniques." Remote Sensing of Environment 160 (2015): 1-14.

[3] Skataric, Maja, Evgeni V. Nikolaev, and Eduardo D. Sontag. "Fundamental limitation of the instantaneous approximation in fold-change detection models." IET systems biology 9, no. 1 (2014).

[4] Banerjee, Taposh, Yu Christine Chen, A. Garcia, and V. Veeravalli. "Power system line outage detection and identification-A quickest change detection approach." In Proceedings of the IEEE International Conference on Acoustics, Speech, and Signal Processing (ICASSP), Florence, Italy, pp. 49. (2014).

[5] Long, Stephanie, Temilola E. Fatoyinbo, and Frederick Policelli. "Flood extent mapping for Namibia using change detection and thresholding with SAR." Environmental Research Letters 9, no. 3 (2014): 035002 .

[6] Kim, Ka Young, et al. "Geometric change of a thiacalix [4] arene supramolecular gel with volatile gases and its chromogenic detection for rapid analysis." Inorganic chemistry 53.6 (2014), pp. 3004-3011.

[7] Nigro, M. B., S. N. Pakzad, and S. Dorvash. "Localized structural damage detection: a change point analysis." Computer-Aided Civil and Infrastructure Engineering 29, no. 6 (2014), pp. 416-432.

[8] Guo, Zhe, et al. "Land cover classification and change detection analysis using LandSat series and geospatial datasets in Nepal from 1980 to 2010." Agro-Geoinformatics (Agro-geoinformatics), 2015 Fourth International Conference on. IEEE, (2015).

[9] Halpern, Joseph Y., and Rafael Pass. "Algorithmic rationality: Game theory with costly computation." Journal of Economic Theory 156 (2015), pp. 246-268.

[10] Tian, Yihui, Kannan Govindan, and Qinghua Zhu. "A system dynamics model based on evolutionary game theory for green supply chain management diffusion among Chinese manufacturers." Journal of Cleaner Production 80 (2014), pp. 96-105.

[11] Peng, Mugen, Yaohua Sun, Chengdan Sun, and Manzoor Ahmed. "Game Theory-Based Radio Resource Optimization in Heterogeneous Small Cell Networks (HSCNs)." Game Theory Framework Applied to Wireless Communication Networks (2015): 137.

[12] Carotenuto, Vincenzo, Antonio De Maio, Carmine Clemente, and John Soraghan. "Invariant rules for multipolarization SAR change detection." Geoscience and Remote Sensing, IEEE Transactions on 53, no. 6 (2015), pp.3294-3311.

[13] Cho, Haeran, and Piotr Fryzlewicz. "Multiple-change-point detection for high dimensional time series via sparsified binary segmentation." Journal of the Royal Statistical Society: Series B (Statistical Methodology) 77, no. 2 (2015), pp. 475-507.

[14] ] Cleynen, Alice, et al. "Fast estimation of the Integrated Completed Likelihood criterion for changepoint detection problems with applications to Next-Generation Sequencing data." Signal Processing 98 (2014), pp. 233-242.

[15] ] Bi, Chujian, Qiushi Zhang, Rui Bao, and Haoxiang Wang. "SAR image restoration and change detection based on game theory." In Intelligent Computing and Internet of Things (ICIT), 2014 International Conference on, pp. 55-58. IEEE, (2015).

[16] Bayraktar, Erhan, and Lifeng Lai. "Byzantine fault tolerant distributed quickest change detection." SIAM Journal on Control and Optimization 53, no. 2 (2015), pp. 575-591.

[17] Akinbobola, A. "Land use/land cover change detection in some selected stations in Anambra State." Journal of Geography and Regional Planning 8, no. 1 (2015) pp. 1-11.

[18] Tsai, Du-Ming, and Ching-Ying Huang. "A motion and image analysis method for automatic detection of estrus and mating behavior in cattle." Computers and Electronics in Agriculture 104 (2014), pp. 2531.

[19] Bi, Chujian, Haoxiang Wang, and Rui Bao. "SAR image change detection using regularized dictionary learning and fuzzy clustering." Cloud Computing and Intelligence Systems (CCIS), 2014 IEEE 3rd International Conference on. IEEE, (2014).

[20] Anandakrishnan, N., and S. Santhosh Baboo. "An Evaluation of Popular Edge Detection Techniques in Digital Image Processing." In Intelligent Computing Applications (ICICA), 2014 International Conference on, pp. 213-217. IEEE, (2014).

[21] ] Dinh, Tran Hiep, Minh Trien Pham, Manh Duong Phung, Due Manh Nguyen, Van Manh Hoang, and Quang Vinh Tran. "Image segmentation based on histogram of depth and an application in driver distraction detection." In Control Automation Robotics \& Vision (ICARCV), 2014 13th International Conference on, pp. 969-974. IEEE, (2014).

[22] Hashemi, Farid, Ahad Kazemi, and Soodabeh Soleymani. "Assessment of an adaptive neuro fuzzy inference system for islanding detection in distributed generation." Journal of Intelligent \& Fuzzy Systems: Applications in Engineering and Technology 26.1 (2014), pp. 19-31. 
[23] Föhst, S., W. Wagner, M. Ackermann, C. Redenbach, K. Schladitz, O. Wirjadi, A. B. Ysasi, S. J. Mentzer, and M. A. Konerding. "Three-dimensional image analytical detection of intussusceptive pillars in murine lung." Journal of microscopy (2015).

[24] Ranallo, Simona, Marianna Rossetti, Kevin W. Plaxco, Alexis Vallée-Bélisle, and Francesco Ricci. "A Modular, DNA-Based Beacon for Single-Step Fluorescence Detection of Antibodies and Other Proteins." Angewandte Chemie (2015).

[25] Senni, L., M. Ricci, A. Palazzi, P. Burrascano, P. Pennisi, and F. Ghirelli. "On-line automatic detection of foreign bodies in biscuits by infrared thermography and image processing." Journal of Food Engineering 128 (2014), pp. 146-156.

[26] Wang, Danzhu, and Maoxiang Lang. "Co-opetition Relationship of Regional Logistics Nodes Based on Evolutionary Game Theory." In LISS 2013, pp. 1325-1331. Springer Berlin Heidelberg, (2015).

[27] Murthy, DN Prabhakar, and Nat Jack. "Introduction to Stochastic Optimisation and Game Theory." In Extended Warranties, Maintenance Service and Lease Contracts, pp. 77-87. Springer London, (2014).

[28] Finus, Michael, and Alejandro Caparros. Handbook on Game Theory and International Environmental Cooperation: Essential Readings. Edward Elgar, (2015).

[29] ] Imran, Ali, Mehdi Bennis, and Lorenza Giupponi. "Use of learning, game theory and optimization as biomimetic approaches for self-organization in macro-femtocell coexistence." Wireless Communications and Networking Conference Workshops (WCNCW), 2012 IEEE. IEEE, (2012).

[30] Abraham, Ittai, Lorenzo Alvisi, and Joseph Y. Halpern. "Distributed computing meets game theory: combining insights from two fields." ACM SIGACT News 42.2 (2011), pp. 69-76.

[31] Mezue, Melvin, et al. "Optimization and reliability of multiple postlabeling delay pseudo-continuous arterial spin labeling during rest and stimulus-induced functional task activation." Journal of Cerebral Blood Flow \& Metabolism (2014).

[32] Davis, James M., Guillermo Gallego, and Huseyin Topaloglu. "Assortment optimization under variants of the nested logit model." Operations Research 62.2 (2014), pp. 250-273.

[33] Bao, Truong Q., and Boris S. Mordukhovich. "Necessary nondomination conditions in set and vector optimization with variable ordering structures." Journal of Optimization Theory and Applications 162.2 (2014), pp. 350-370.

[34] Farasat, Iman, et al. "Efficient search, mapping, and optimization of multi-protein genetic systems in diverse bacteria." Molecular systems biology 10.6 (2014): 731.

[35] Posa, Michael, Cecilia Cantu, and Russ Tedrake. "A direct method for trajectory optimization of rigid bodies through contact." The International Journal of Robotics Research 33.1 (2014), pp. 69-81.

[36] Heigold, Georg, et al. "Asynchronous stochastic optimization for sequence training of deep neural networks." Acoustics, Speech and Signal Processing (ICASSP), 2014 IEEE International Conference on. IEEE, (2014).

[37] ] Lijesh, K. P., and Harish Hirani. "Development of Analytical Equations for Design and Optimization of Axially Polarized Radial Passive Magnetic Bearing." Journal of Tribology 137.1 (2015): 011103.

[38] Sierzenski, Paul R., et al. "Applications of justification and optimization in medical imaging: examples of clinical guidance for computed tomography use in emergency medicine." Journal of the American College of Radiology 11.1 (2014), pp. 36-44.

[39] Eichfelder, Gabriele. "Numerical procedures in multiobjective optimization with variable ordering structures." Journal of Optimization Theory and Applications 162.2 (2014), pp. 489-514.

[40] Li, Miqing, Shengxiang Yang, and Xiaohui Liu. "Shift-based density estimation for Pareto-based algorithms in many-objective optimization." Evolutionary Computation, IEEE Transactions on 18.3 (2014), 